\title{
On making sense of neural networks in road analysis
}

\author{
Daniel Morris \\ Faculty of Engineering and \\ Physical Sciences \\ University of Surrey \\ Guildford, Surrey \\ Email:dm00247@surrey.ac.uk
}

\author{
Andreas Antoniades \\ Faculty of Engineering and \\ Physical Sciences \\ University of Surrey \\ Guildford, Surrey \\ Email:a.antoniades@surrey.ac.uk
}

\author{
Clive Cheong Took \\ Faculty of Engineering and \\ Physical Sciences \\ University of Surrey \\ Guildford, Surrey \\ Email: c.cheongtook@surrey.ac.uk
}

\begin{abstract}
Neural networks have been treated as "black boxes" for the majority of the machine learning community. The difficulty in making sense of neural networks lies in the complex topology of the hidden layers. Although there have been works in the literature aimed at demystifying the way neural networks operate, making sense of the hidden layer still remains a challenge. In this work, we propose a way to derive physical meaning from the hidden layer by mapping our neural network to the topology of a Bayesian network. Using this mapping, we enhance the probabilities of the Bayesian network resulting in a hybrid model that outperforms both the Bayesian and neural networks in the task of traffic accident prediction. Our analysis suggests that a neural network can estimate the node probabilities of a Bayesian network if mapped accordingly.
\end{abstract}

\section{Introduction}

The past decade was marked with great successes by neural network (NN) models [1]-[3] attaining close to human accuracy. Despite these achievements, NNs remain relative "black boxes" in nature to both scientists and analysts [4]. The difficulty in deriving physical meaning out of these statistical models lies in the complexity of the hidden layer structure. To some extent, deep learning has addressed this issue, as it has been shown that each hidden layer learns a particular feature of the dataset. In contrast to deep learning, this physical interpretation does not apply to traditional NNs in the form of shallow learning, for there may not be adequate number of hidden layers to represent each feature of the dataset. This paper addresses this shortcoming in the literature.

There have been attempts in the literature to study the inner workings of neural networks. An early work looked at establishing the equivalence ( $f$-duality) between a certain class of neural networks and fuzzy rule based systems [4]. A more recent work investigated the effect of groups of inputs (and not hidden neurons) on the output of a deep learning neural network [5]. These works provided valuable insights, yet it is still not clear on the role and contribution of each individual neuron in the hidden layer of a shallow neural network. One way to make sense of neural networks is to make use of Bayesian networks (BNs). In contrast to neural networks, Bayesian networks illustrate clearly the relationship between various nodes (neurons), and it is straightforward to have a physical interpretation of the network. Yet, the relationship between a Bayesian network and a neural network is still not clear. Motomura and Hara modelled the inputs of a $\mathrm{NN}$ to the source nodes of a $\mathrm{BN}$, the hidden neurons of $\mathrm{NN}$ as the intermediate node of a $\mathrm{BN}$, and the output neurons of a $\mathrm{NN}$ as the output node of BN [6]. Although, this modelling makes sense in terms of the flow of information, it does not illustrate how this mapping of a $\mathrm{NN}$ onto a $\mathrm{BN}$ is justified either empirically or theoretically. On the other hand, Richard and Lippmann demonstrated theoretically that it was possible to learn the posterior probabilities of a BN using a neural network by adapting the latter's cost function [7]

$$
J=\sum_{j} \sum_{i}\left[y_{i}(\mathbf{x})-d_{i}\right]^{2} p\left(\mathbf{x}, c_{j}\right)
$$

where $\mathbf{x}, y_{i}, d_{i}$, and $p\left(\mathbf{x}, c_{j}\right)$ denotes the input, the $i t h$ output and desired output, and the joint probability between the input and the $j$ th class. Although it is useful to have the NN estimate the probabilities for the BN, the adapted cost function (1) is different from a traditional neural network. Therefore, this work did not shed light on the learning operation of a traditional neural network.

Our contribution is three fold. First, a mapping algorithm is proposed to translate from a $\mathrm{BN}$ to a NN. The mapping algorithm enables us to deduct the physical meaning of each hidden neuron. We then show that the probabilities of a BN follow similar trends as those of the weights of the $\mathrm{NN}$; this confirms that each particular hidden neuron corresponds to a particular feature in the dataset. Second, we demonstrate how the weights of a NN can improve the estimated probabilities associated with a BN. Third, to give context to our work, we illustrate how this collaborative mechanism between $\mathrm{BN}$ and $\mathrm{NN}$ can be useful in road accident analysis. 


\section{Road Accident Analysis}

For road accident analysis, there have been many problems considered in IJCNN ranging from traffic incident detection using Neuro-Fuzzy networks [8] to the prediction of "near-misses" using Hidden Markov Models [9]. Whilst these approaches have the potential to predict accidents, the conditions upon which each accident occurs are not identified and focused instead on estimating the severity of a given accident provided with distinct patterns of variables. The problem considered in this work is focused instead on forecasting the total outcomes of accidents.

\subsection{Accident Data Set}

The data contained a vast variety of variables, collected by UK police at the scene of a collision in Kent UK. Initially, the key factors involved in road collisions were identified, similarly to [10], and are summarised below.

- Weather $(\mathrm{W})$ : The weather on the road will have an effect on the visibility of other cars to a given driver, in addition to having a knock-on effect upon the road surface in the event of adverse weather such as rain or snow.

- Road Surface (RS): The surface of a road will have a large effect on a vehicle's stopping distance, in addition to potential accidents which may occur as a result of losing grip upon the road, such as skidding.

- Lighting (L): The lighting of the road, which largely effects the visibility of other road users and pedestrians. This will be effected by the time of day

- Time (T): Different numbers of cars use roads at different times, as a result accidents rates will be affected. Whilst the effect of night versus day may be cancelled out by other factors, the impact of time upon accident rates must be considered in the model.

- Speed of Road (S): An increase in the speed of a given road will increase the distance a vehicle travels in a given amount of time, and as such will increase both the thinking and stopping distance of a given vehicle, and as such will have an effect on accident rates. It may also be observed that at different speeds, road users are more inclined to overtake other road users travelling below the speed limit, or may be unduly careless at lower speeds.

- Flow Rate of Road (F): Refers to the number of vehicles travelling through a road in a given time period. The number of cars travelling through a road will scale the number of accidents likely to happen upon the road, however this is not a linear relationship.
TABLE 1: Nodes and associated states

\begin{tabular}{|c|c|c|}
\hline Node Label & State $=0$ & State $=1$ \\
\hline Flow & Low & High \\
Lighting & Bad & Good \\
Road Surface & Poor & Good \\
Weather & Rain/Other & Sun \\
Speed & $<65 \mathrm{kmph}$ & $\geq 65 \mathrm{kmph}$ \\
Time & Night & Day \\
Accident & True & False \\
\hline
\end{tabular}

Identifying the effects these variables have upon accidents rates involved data mining, in order to determine both the probabilities of these events, and the effect they have on accident rates. Due to the relatively small size of the dataset, all relationships and states are reduced to binary form, the nodes and their associated binary states ${ }^{1}$ are shown in Table 1. This resulted in 64 different combinations of binary states possible for an accident to occur in. Those accidents which did not have information for every state were filtered out for purposes of accuracy in training. The resulting dataset was reduced to 3312 accidents across 66 roads.

\subsection{Bayesian network for accident analysis}

A Bayesian network models a set of random variables and their conditional dependencies in a directed acyclic graph. In the context of our work, the Bayesian network represents the probabilistic relationships between accident causes and accidents. While the initial training of the Bayesian network requires expert knowledge of the data in order to generate the initial probabilities, this would apply to most fields in which a Bayesian network is used. Given accident causes, the network can be used to forecast whether an accident will happen or not. The key variables and their effect on other variables discussed in Section 2.1 were used as nodes for the Bayesian network as illustrated in Fig. 1. For example, the joint probability of having an accident $(\mathrm{A}=1)$ when it is night $(\mathrm{T}=0)$, dark $(\mathrm{L}=0)$, a low speed accident $(\mathrm{S}=0)$ on a good road surface $(\mathrm{RS}=1)$ and good weather $(\mathrm{W}=1)$, when there is a low flow rate of vehicles $(\mathrm{F}=0)$ is given by

$P[A=1, T=0, L=0, S=0, R S=1, W=1, F=0]=$

$P[A=1 \mid T=0, L=0, S=0, R S=1, W=1, F=0] P[T=0] \times$

$P[L=0 \mid T=0] P[S=0] P[R S=1 \mid W=1] P[W=1] P[F=0]$

There are 64 such probabilities of having an accident based on the six accident factors described in Section 2.1.

\section{Mapping Bayesian Networks to Neural Net- works}

To map the hidden neurons to the nodes of the Bayesian network, the number of hidden neurons of the neural network was set to 12 as there are two states for each of the six accident factors. The topology of the neural network employed

1. For the purpose of the neural network, all 0 states were changed to -1 to prevent 0 weights cascading through the network. 


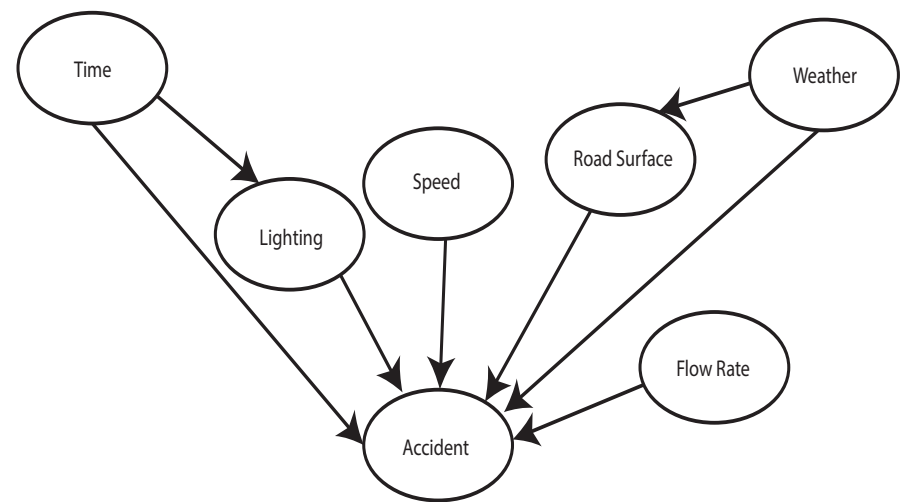

Figure 1: Bayesian Network

is shown in Fig. 3, with input neurons $\mathbf{x}$, hidden neurons $\mathbf{y}=\phi(\mathbf{W x})$, and the output neuron $z=\mathbf{v}^{T} \mathbf{y}$, where $\phi(\cdot)$ denotes the nonlinear activation function and $\mathbf{W}$ and $\mathbf{v}$ are the weight coefficients for the hidden layer and the output layer respectively, a feedforward multilayer perceptron was used for this problem. The weights of the NN were randomly initialised, and trained against the training data using back propagation.

The next step was to establish a similarity mechanism between $\mathrm{NN}$ and $\mathrm{BN}$. It is not uncommon to consider the weights of the input layer of a neural network as proxies of the influence of input variables [11][12]. This way, a hidden neuron can be identified to correspond to a particular accident factor (neural network input). In particular, the labelling of the $i$ th hidden neuron can be performed as follows:

1) Assign label $\left(y_{i}\right)=\operatorname{label}\left(x_{j}\right)$ if $\max _{i, j}(|\mathbf{W}|)$

2) Set all weight coefficients to zero that feed into $i$ th hidden neuron $y_{i}$, i.e. $w_{i, j}=0 \quad \forall j$

3) If label $\left(x_{j}\right)=\operatorname{label}\left(y_{i}\right)=\operatorname{label}\left(y_{k}\right)$, where $i \neq k$, then set all weight coefficients to zero that comes out of the $j$ th input neuron $x_{j}$, i.e. $w_{i, j}=0 \quad \forall i$

Step 1 ensures that hidden neuron $y_{i}$ has the same label as $x_{j}$, since the link between this pair of neurons is strongest. For example, if label $\left(x_{j}\right)$ is the accident factor 'speed', then the label of the hidden neuron $y_{i}$ also corresponds to speed. Step 2 and 3 guarantee that a hidden neuron does not get labelled more than once and the label of an input neuron is not used more than twice for the hidden neurons, as each accident factor has only two states (either 0 or 1). This process iterates for all hidden neurons. To demonstrate the validity of the labelling process in Step 1-3, Fig. 2 compares the ratios between the pairwise probabilities of $\mathrm{BN}$ with the ratios between the pairwise output weight coefficients $\mathbf{v}$ in NN for different accident factors. Thus, we assign hidden neurons to the input neurons of the $\mathrm{NN}$ in a way that reflects the structure of the $\mathrm{BN}$.

- Remark 1: Fig. 2 shows that four out of six accident ratio factors for both $\mathrm{BN}$ and $\mathrm{NN}$ were quite close, i.e. for speed, weather, lighting, surface. This

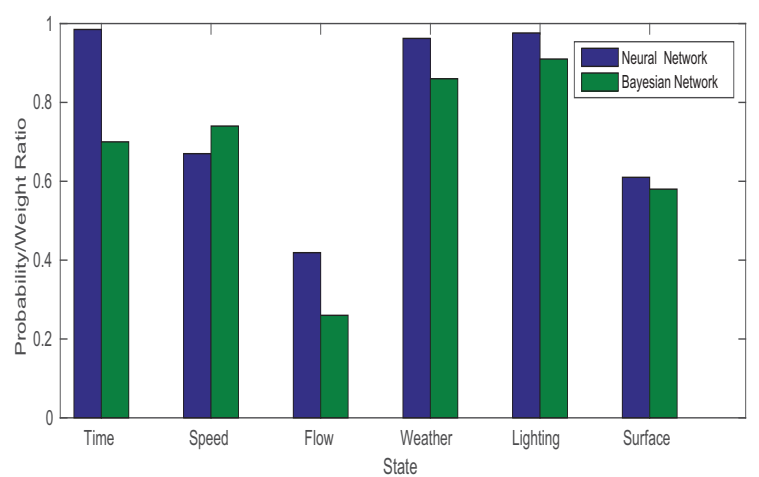

Figure 2: Similarity between Bayesian and Neural Network

indicates that labelling process in Step 1-3 was reasonable.

- Remark 2: Although there is a visible difference in ratios for flow, both ratios were lowest compared to the other ratios. This again confirms that Step 1-3 of the labelling process of the hidden neurons was reasonable.

- Remark 3: Fig. 2 indicates that the factors time, weather, and lighting were the least influential factors in an accident due to the proximity of the ratio to unity (e.g. the probability of an accident happening during day is similar to the probability of an accident occurring during night). Conversely, the factors speed, flow, and road surface played an important role in an accident occurring.

- Remark 4: The only factor in which both the Bayesian network and the neural network did not concur was time. On one hand, the neural network indicates that time is not a deciding factor in an accident (ratio $\approx 1$ ), whereas the Bayesian network indicates otherwise.

2 Now that each accident factor can be associated with a pair of hidden neurons based on Step 1-3, the problem lies in identifying which of the two hidden neurons corresponds to which state of the accident factor. For instance, if the accident factor is 'flow', then one of the hidden neuron should correspond to low flow, and the other hidden neuron to high flow. The state mapping problem can be be solved by corresponding the high state probability of $\mathrm{BN}$ with the high weight coefficient $v_{i}$ of $\mathrm{NN}$ as follows:

$$
\begin{aligned}
\operatorname{state}\left(y_{i}\right) & =0 & & \text { if } v_{i} \geq v_{j} \& P\left[\operatorname{label}\left(y_{i}\right)=0\right] \geq P\left[\operatorname{label}\left(y_{j}\right)=1\right] \\
& =1 & & \text { otherwise }
\end{aligned}
$$

provided label $\left(y_{i}\right)=\operatorname{label}\left(y_{j}\right)$ and $i \neq j$, where $P[\cdot]$ is the probability obtained from the Bayesian network, and $v_{i}$ is the weight of the output neuron. For more details on the different states, see Table 1. Step 1-3 along with Equation

2. The state mapping in Equation (2) can be extended to match features that have more than 2 states by ranking the weights based on their magnitude and the states based on the norm of their probabilities. 


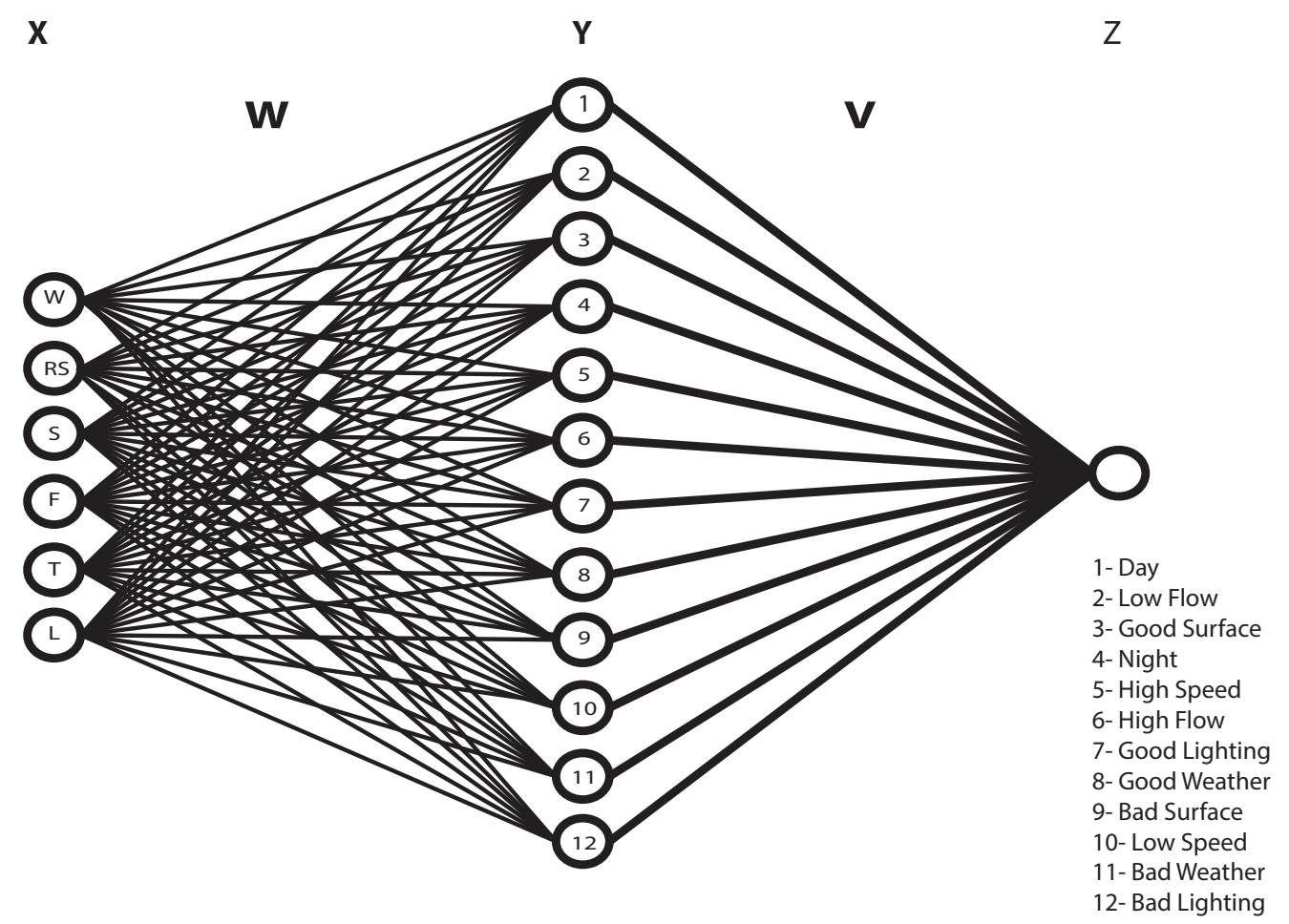

Figure 3: Making sense of hidden neurons in neural networks in road analysis. The inputs were weather (W), Road Surface (RS), Lighting (L), Time (T), Speed of Road (S), Flow Rate of Road (F).

(2) enable us to determine the physical meaning of each hidden neuron of a neural network by making use of a Bayesian network.

Remark 4 highlights the need for a collaborative mechanism between a Bayesian network and a neural network so that if one model fails to capture important features from the dataset, the other model can compensate for that loss in information; this is analogous to ensemble learning. The Bayesian network directly imprints information from the data in its structure, on the other hand the neural network learns an approximation function that generalises observations from the data. This resulted in the BN returning better results at high accident rates and the $\mathrm{NN}$ returning better results at low accident rates. We therefore propose a collaborative mechanism based on the key observation that the ratios shown in Fig. 2 can be used to improve the learning accuracy of the 'inferior' model. For a given accident factor (e.g. flow), consider the ratios of $\mathrm{NN}$ and $\mathrm{BN}$ respectively as

$$
\begin{aligned}
\kappa_{N} & =\frac{\min \left(v_{i}, v_{j}\right)}{\max \left(v_{i}, v_{j}\right)} \\
\kappa_{B} & =\frac{\min \left(P\left[\operatorname{label}\left(y_{i}\right)\right]\right)}{\max \left(P\left[\operatorname{label}\left(y_{i}\right)\right]\right)}
\end{aligned}
$$

assuming that label $\left(y_{i}\right)=\operatorname{label}\left(y_{j}\right)$. If the training error
$\mathrm{E}(\mathrm{BN}) \geq \mathrm{E}(\mathrm{NN})$, then we need to update the probabilities of $\mathrm{BN}$ using the weight coefficients $\mathbf{v}$ of $\mathrm{NN}$ as follows. First, set the ratio of the probabilities of $\mathrm{BN}$ as $\kappa_{N}$ such that

$$
\min \left(P\left[\operatorname{label}\left(y_{i}\right)\right]\right)+\max \left(P\left[\operatorname{label}\left(y_{i}\right)\right]\right)=1
$$

Equation (5) allows us to determine the two probabilities associated an accident factor, since

$$
\begin{aligned}
\max \left(P\left[\operatorname{label}\left(y_{i}\right)\right]\right) & =\frac{1}{\kappa_{N}+1} \\
\min \left(P\left[\operatorname{label}\left(y_{i}\right)\right]\right) & =1-\max \left(P\left[\operatorname{label}\left(y_{i}\right)\right]\right)
\end{aligned}
$$

These estimated probabilities are adequate for unconditional probabilities such as for accident factor time, however, these are not adequate for determining conditional probabilities such as for accident factor lighting. In such a case, these conditional probabilities can be approximated, when $\operatorname{label}\left(y_{i}\right) \neq \operatorname{label}\left(y_{j}\right)$ as

$$
P\left[\operatorname{label}\left(y_{i}\right) \mid \operatorname{label}\left(y_{j}\right)\right] \approx P\left[\operatorname{label}\left(y_{i}\right)\right] P\left[\operatorname{label}\left(y_{j}\right)\right]
$$

Otherwise if training error $\mathrm{E}(\mathrm{BN})<\mathrm{E}(\mathrm{NN})$, the weight coefficients of the neural network can be updated such that

$$
\min \left(v_{i}, v_{j}\right)=\kappa_{B} \max \left(v_{i}, v_{j}\right)
$$

The algorithm is described in Algorithm 1. 
In order to measure the error of the models, we utilised Chebyshev distance, calculated by Equation (10). Chebyshev distance between two vectors, in this case the predicted and actual number of accidents, is the greatest distance in any state.

$$
\begin{aligned}
E:(\mathbf{x}, \mathbf{y}) \rightarrow\|\mathbf{x}-\mathbf{y}\|_{\infty} & =\lim _{p \rightarrow \infty}\left(\sum_{i=1}^{n}\left|x_{i}-y_{i}\right|^{p}\right)^{\frac{1}{p}} \\
& =\max _{i}\left|x_{i}-y_{i}\right|
\end{aligned}
$$

where $\mathbf{x}$ and $\mathbf{y}$ are vectors and $\|\cdot\|$ is the norm of a vector.

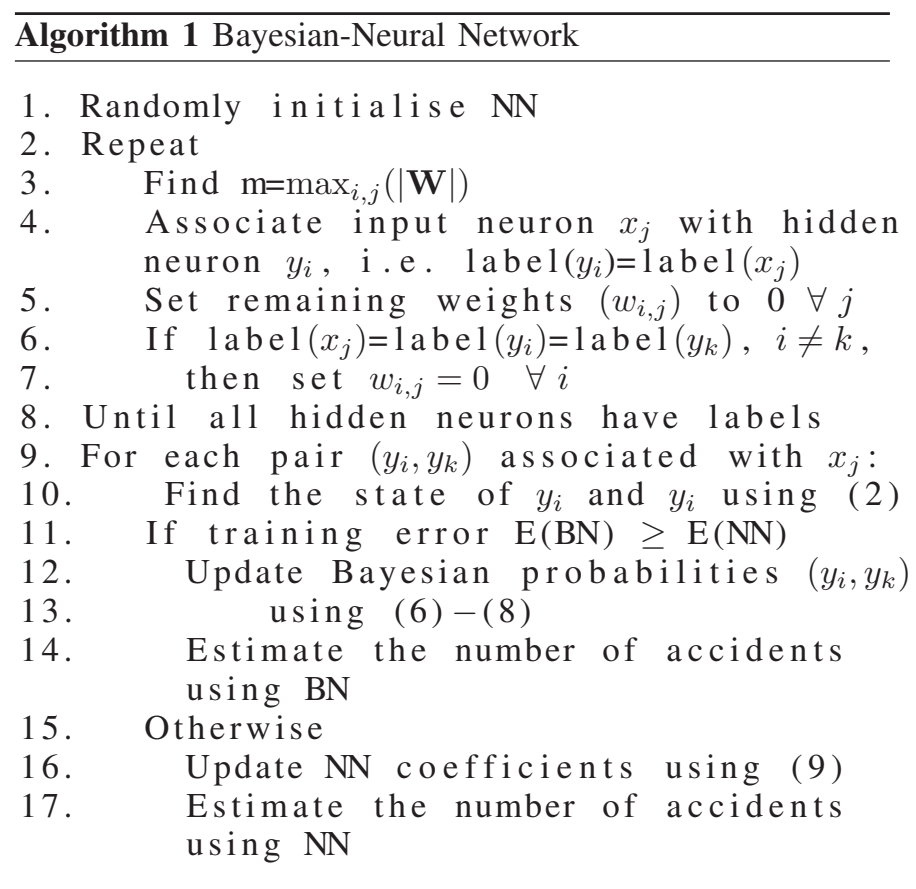

\section{Simulations}

For the purpose of the simulation, the data described in Section 2.1 was split into a training and a testing set, with years from 2005-2013 being placed into the training set, and 2013-2015 being the testing set, resulting in training set and testing sets of 2946 and 366 respectively. Crossvalidation was not considered as the data is presented as a time series. The proposed Bayesian-Neural collaborative network described in Algorithm 1 was compared against the Bayesian network and the neural network on forecasting the number of accidents in given states. There are for 64 different states based on the six accident factors shown in Table 1. Fig. 4 shows that forecasted number of accidents by the three algorithms and the actual number of accidents based on the 64 states. Table 2 summarises the overall performance of the three algorithms.

- Remark 5: From the two left subplots of Fig. 4, the neural network provided better estimates, when the number of accidents was low (e.g. in State 34), whereas the Bayesian network provided better estimates when there were a high number of accidents (e.g. in State 42). As the occurrence of low number of accidents exceeded the occurrence of high number of accidents, the neural network outperformed the Bayesian network. This is confirmed by the overall accuracy shown in Table 2.

- Remark 6: The proposed Bayesian-Neural network benefited from the ensemble learning of both the neural network and the Bayesian network; this was clear from the enhanced performance over the latter across the 64 states, as shown in Fig. 4. This was also confirmed by the overall performance accuracy shown in Table 2.

TABLE 2: Overall Accuracy of networks

\begin{tabular}{|c|c|}
\hline Model & Error (E) \\
\hline Neural Network & 32.29 \\
Bayesian Network & 40.00 \\
Bayesian-Neural Network & 15.00 \\
\hline
\end{tabular}

\section{Conclusion}

To address the 'black-box' nature of neural networks, we have shown how hidden neurons can have physical meaning in the learning process of neural networks. In particular, it has been shown how each hidden neuron can be associated with accident causes in the context of road analysis by making use of a Bayesian network. As a side product, a collaborative mechanism between a Bayesian network and a neural network was proposed to improve on the accuracy of the forecasted accidents. Indeed, ensemble learning of the proposed Bayesian-Neural network leveraged the ability of the neural networks to predict the general trend of data with low variance, and the ability of Bayesian networks to forecast 'outlier' data of high variance to improve the accuracy in terms of number of accidents.

\section{References}

[1] C. Szegedy et al., "Going deeper with convolutions", in Proc. IEEE Conf. CVPR, pp. 1-9, Jun. 2015.

[2] G. E. Hinton and R. Salakhutdinov, "Reducing the dimensionality of data with neural networks", Science, vol. 313, no. 5786, pp. 504-507, May 2006.

[3] I. Goodfellow, J. Pouget-Abadie, M. Mirza, B. Xu, D. Warde-Farley, S. Ozair, A. Courville, and Y. Bengio. "Generative adversarial nets", NIPS, pp. 2672-2680, Jun. 2014.

[4] J. M. Benitez, J. L. Castro and I.Requena, "Are Artificial Neural Networks Black Boxes?”, IEEE Trans. on Neural Networks, vol. 8, no. 5, pp. 1156-1164, Sept. 1997.

[5] W. Samek, A. Binder, G. Montavon, S. Bach, and K. R. Muller, "Evaluating the visualization of what a deep neural network has learned," CoRR, vol. abs/1509.06321, Sept. 2015.

[6] Y. Motomura and I. Hara, "Bayesian Network Learning System based on Neural Networks", International Symposium on Theory and Applications of Soft Computing, 2000. 

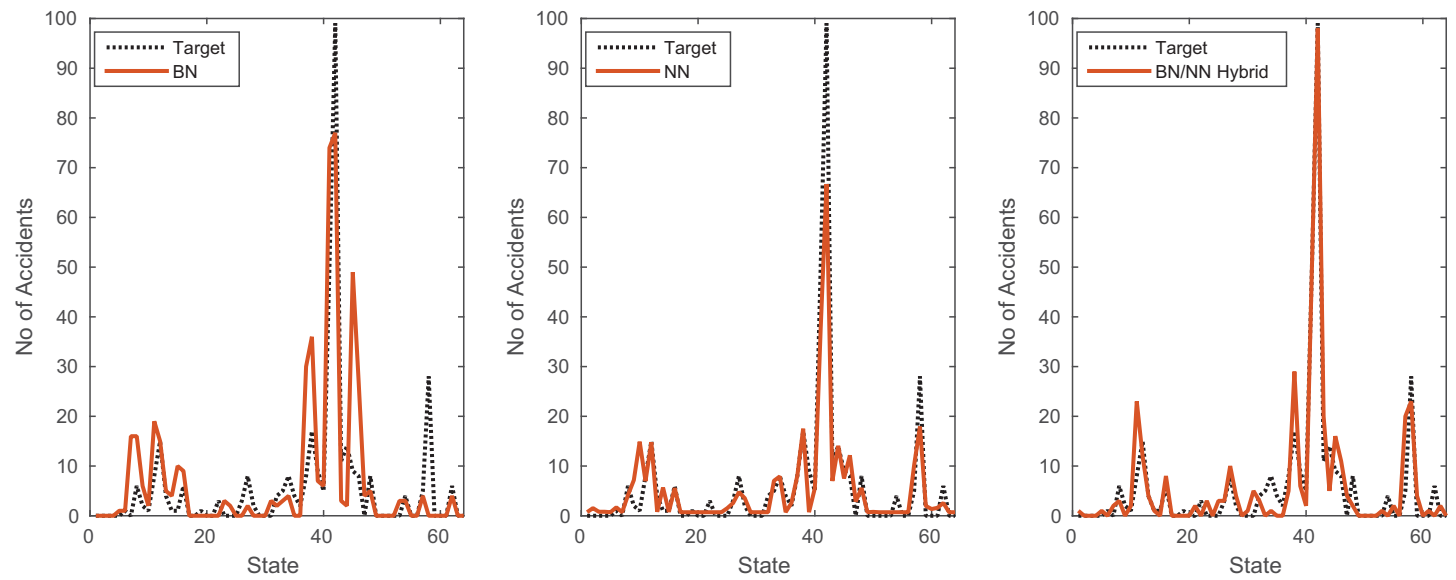

Figure 4: Left to Right: Bayesian Network, Neural Network, Bayesian-Neural Network predictions for accident rates in each of the 64 states

[8] D. Srinivasan, S. Sanyal, and T. W. Wan, "Hybrid Neuro-Fuzzy Technique for Automated Traffic Incident Detection," International Joint Conference on Neural Networks, pp. 713-719, Vancouver 2006.

[9] N. Saunier and T. Sayed, "Clustering Vehicle Trajectories with Hidden Markov Models. Application to Automated Traffic Safety Analysis", International Joint Conference on Neural Networks, pp. 4132-4138, Vancouver 2006.

[10] T. Tambouratzis, D. Souliou, M. Chalikias and A. Gregoriades, "Combining Probabilistic Neural Networks and Decision Trees for Maximally Accurate and Efficient Accident Prediction”, Jul. 2010.

[11] A. Antoniades and C. C. Took, "Speeding Up Feature Selection: A Deep-Inspired Network Pruning Algorithm", International Joint Conference on Neural Networks, Vancouver 2016.

[12] R. Tibshirani, "Regression Shrinkage and Selection via the Lasso", Journal of the Royal Statistical Society, vol. 58, no. 1, pp. 267-288, Jan. 1995.
[7] M. Richard and R. Lippmann, "Neural network classifiers estimate Bayesian a posteriori probabilities", Neural Computation vol. 3, no. 4, pp. 461-483, 1991. 